\title{
ERRATUM
}

\section{Fetecau - T. Hayat - M. Khan - Corina Fetecau \\ Erratum to: Unsteady flow of an Oldroyd-B fluid induced by the impulsive motion of a plate between two side walls perpendicular to the plate}

Published online: 16 November 2010

(C) Springer-Verlag 2010

Erratum to: Acta Mech 198 (2008) 21-33

DOI 10.1007/s00707-007-0522-0

\section{Introduction}

In a recent paper [1], the unsteady flow of an Oldroyd-B fluid induced by the impulsive motion of a flat plate between two side walls perpendicular to the plate has been studied using the Fourier sine transform. It is found that in view of omission of a critical term from the resulting subsidiary equation, the derived solutions are incomplete.

The aim of this note is to correct the previous analysis and to give the complete solutions corresponding to this motion. Employing the same notation convention as used in [1], we begin by restating the initial-boundary value problem

$$
\begin{gathered}
\lambda \partial_{\mathrm{t}}^{2} \mathrm{u}(\mathrm{y}, \mathrm{z}, \mathrm{t})+\partial_{\mathrm{t}} \mathrm{u}(\mathrm{y}, \mathrm{z}, \mathrm{t})=v\left(1+\lambda_{\mathrm{r}} \partial_{\mathrm{t}}\right)\left(\partial_{\mathrm{y}}^{2}+\partial_{\mathrm{z}}^{2}\right) \mathrm{u}(\mathrm{y}, \mathrm{z}, \mathrm{t})=0 ; \mathrm{y}, \mathrm{t}>0, \mathrm{z} \in(0, \mathrm{~d}) \\
\mathrm{u}(\mathrm{y}, \mathrm{z}, 0)=\partial_{\mathrm{t}} \mathrm{u}(\mathrm{y}, \mathrm{z}, 0)=0 \text { for } \mathrm{y}>0 \text { and } \mathrm{z} \in[0, \mathrm{~d}] \\
\mathrm{u}(0, \mathrm{z}, \mathrm{t})=\mathrm{V} \text { for } \mathrm{t}>0, \mathrm{z} \in(0, \mathrm{~d}) ; \mathrm{u}(\mathrm{y}, 0, \mathrm{t})=\mathrm{u}(\mathrm{y}, \mathrm{d}, \mathrm{t})=0 \text { for } \mathrm{y}, \mathrm{t}>0 \\
\mathrm{u}(\mathrm{y}, \mathrm{z}, \mathrm{t}), \partial_{\mathrm{y}} \mathrm{u}(\mathrm{y}, \mathrm{z}, \mathrm{t}) \rightarrow 0 \text { as } \mathrm{y} \rightarrow \infty, \mathrm{z} \in[0, \mathrm{~d}] \text { and } \mathrm{t}>0
\end{gathered}
$$

In order to solve the linear partial differential Eq. (1) with the initial and boundary conditions (2)-(4), the Fourier sine and Laplace transforms are used.

The online version of the original article can be found at doi:10.1007/s00707-007-0522-0.

C. Fetecau $(\varangle)$

Department of Mathematics, Technical University of Iasi, Iasi 700050, Romania

E-mail: fetecau_constantin@yahoo.com

Tel.: +40-32-263218

T. Hayat $\cdot$ M. Khan

Department of Mathematics, Quaid-i-Azam University, Islamabad 45320, Pakistan

T. Hayat

Department of Mathematics, King Saud University, Riyadh 11451, Saudi Arabia

Corina Fetecau

Department of Theoretical Mechanics, Technical University of Iasi, Iasi 700050, Romania 


\section{Corrected solutions}

For the sake of generality we start the problem with the boundary condition $\mathrm{u}(0, \mathrm{z}, \mathrm{t})=\mathrm{V}(\mathrm{t})$ where $\mathrm{V}(0)=0$. Taking $\mathrm{V}(\mathrm{t})=\mathrm{VH}(\mathrm{t})$, where $\mathrm{V}$ is a constant and $\mathrm{H}(\mathrm{t})$ is the Heaviside step function, the solution of our problem will be obtained, while for $\mathrm{V}(\mathrm{t})=\mathrm{V} \cos (\omega \mathrm{t})$ or $\mathrm{V}(\mathrm{t})=\mathrm{Vt}$ the solutions corresponding to the motion induced by an oscillating or a constantly accelerating plate are recovered. Multiplying Eq. (1) by $\sqrt{2 / \pi} \sin (\mathrm{y} \xi) \sin \left(\lambda_{\mathrm{n}} \mathrm{z}\right)$, integrating the result with respect to $\mathrm{y}$ and $\mathrm{z}$ from 0 to infinity and 0 to $\mathrm{d}$, respectively and taking into account the initial and boundary conditions (2)-(4), we attain the linear differential equation

$$
\begin{gathered}
\lambda \frac{\partial^{2} \mathrm{u}_{\mathrm{sn}}(\xi, \mathrm{t})}{\partial \mathrm{t}^{2}}+\left[1+\alpha\left(\xi^{2}+\lambda_{\mathrm{n}}^{2}\right)\right] \frac{\partial \mathrm{u}_{\mathrm{sn}}(\xi, \mathrm{t})}{\partial \mathrm{t}}+v\left(\xi^{2}+\lambda_{\mathrm{n}}^{2}\right) \mathrm{u}_{\mathrm{sn}}(\xi, \mathrm{t}) \\
=\xi \sqrt{\frac{2}{\pi}} \frac{1-(-1)^{\mathrm{n}}}{\lambda_{\mathrm{n}}}\left[v \mathrm{~V}(\mathrm{t})+\alpha \mathrm{V}^{\prime}(\mathrm{t})\right],
\end{gathered}
$$

where $\lambda_{\mathrm{n}}=\mathrm{n} \pi / \mathrm{d}$ and the double Fourier sine transforms $\mathrm{u}_{\mathrm{sn}}(\xi, \mathrm{t})$ of $\mathrm{u}(\mathrm{y}, \mathrm{z}, \mathrm{t})$ have to satisfy the initial conditions

$$
\mathrm{u}_{\mathrm{sn}}(\xi, 0)=\frac{\partial \mathrm{u}_{\mathrm{sn}}(\xi, 0)}{\partial \mathrm{t}}=0 \text { for } \xi>0 \text { and } \mathrm{n}=1,2,3, \ldots
$$

Applying the Laplace transform to Eq. (5) and using the initial conditions (6), we find

$$
\overline{\mathrm{u}}_{\mathrm{sn}}(\xi, \mathrm{q})=\xi \sqrt{\frac{2}{\pi}} \frac{1-(-1)^{\mathrm{n}}}{\lambda_{\mathrm{n}}} \frac{\alpha \mathrm{q}+v}{\lambda \mathrm{q}^{2}+\left[1+\alpha\left(\xi^{2}+\lambda_{\mathrm{n}}^{2}\right)\right] \mathrm{q}+v\left(\xi^{2}+\lambda_{\mathrm{n}}^{2}\right)} \overline{\mathrm{V}}(\mathrm{q}),
$$

where $\mathrm{q}$ is the transform parameter while $\overline{\mathrm{u}}_{\mathrm{sn}}(\xi, \mathrm{q})$ and $\overline{\mathrm{V}}(\mathrm{q})$ are the Laplace transforms of $\mathrm{u}_{\mathrm{sn}}(\xi, \mathrm{t})$ and $\mathrm{V}(\mathrm{t})$, respectively. If $\mathrm{V}(\mathrm{t})=\mathrm{VH}(\mathrm{t})$ then $\overline{\mathrm{V}}(\mathrm{q})=1 / \mathrm{q}$ and therefore $\overline{\mathrm{u}}_{\mathrm{sn}}(\xi, \mathrm{q})$ can be written in the simple form

$$
\begin{gathered}
\overline{\mathrm{u}}_{\mathrm{sn}}(\xi, \mathrm{q})=\frac{\mathrm{V} \xi}{\lambda} \sqrt{\frac{2}{\pi} \frac{1-(-1)^{\mathrm{n}}}{\lambda_{\mathrm{n}}}} \frac{1}{\mathrm{q}} \mathrm{G}(\xi, \mathrm{q}), \\
\mathrm{G}(\xi, \mathrm{q})=\frac{1}{\mathrm{q}^{2}+\left[1+\alpha\left(\xi^{2}+\lambda_{\mathrm{n}}^{2}\right)\right] \mathrm{q} / \lambda+v\left(\xi^{2}+\lambda_{\mathrm{n}}^{2}\right) / \lambda}=\frac{1}{\mathrm{r}_{2 \mathrm{n}}-\mathrm{r}_{1 \mathrm{n}}}\left(\frac{\alpha \mathrm{r}_{2 \mathrm{n}}+v}{\mathrm{q}-\mathrm{r}_{2 \mathrm{n}}}-\frac{\alpha \mathrm{r}_{1 \mathrm{n}}+v}{\mathrm{q}-\mathrm{r}_{1 \mathrm{n}}}\right), \\
\mathrm{r}_{1 \mathrm{n}}, \mathrm{r}_{2 \mathrm{n}}=\frac{-\left[1+\alpha\left(\xi^{2}+\lambda_{\mathrm{n}}^{2}\right)\right] \pm \sqrt{\left[1+\alpha\left(\xi^{2}+\lambda_{\mathrm{n}}^{2}\right)\right]^{2}-4 v \lambda\left(\xi^{2}+\lambda_{\mathrm{n}}^{2}\right)}}{2 \lambda} .
\end{gathered}
$$

Applying the inverse Laplace transform to Eq. (8) and using the convolution theorem, we obtain

$$
\mathrm{u}_{\mathrm{sn}}(\xi, \mathrm{t})=\frac{\mathrm{V} \xi}{\lambda} \sqrt{\frac{2}{\pi}} \frac{1-(-1)^{\mathrm{n}}}{\lambda_{\mathrm{n}}} \int_{0}^{\mathrm{t}} \mathrm{g}(\xi, \mathrm{s}) \mathrm{ds},
$$

where

$$
\mathrm{g}(\xi, \mathrm{t})=\frac{1}{\mathrm{r}_{2 \mathrm{n}}-\mathrm{r}_{1 \mathrm{n}}}\left[\left(\alpha \mathrm{r}_{2 \mathrm{n}}+v\right) \mathrm{e}^{\mathrm{r}_{2 \mathrm{n}} \mathrm{t}}-\left(\alpha \mathrm{r}_{1 \mathrm{n}}+v\right) \mathrm{e}^{\mathrm{r}_{1 \mathrm{n}} \mathrm{t}}\right]
$$

is the inverse Laplace transform of $\mathrm{G}(\xi, q)$. Direct computations show that Eqs. (9) and (10) imply

$$
\mathrm{u}_{\mathrm{sn}}(\xi, \mathrm{t})=\mathrm{V} \xi \sqrt{\frac{2}{\pi}} \frac{1-(-1)^{\mathrm{n}}}{\lambda_{\mathrm{n}}}\left[\frac{1}{\xi^{2}+\lambda_{\mathrm{n}}^{2}}-\frac{1}{\xi^{2}+\lambda_{\mathrm{n}}^{2}} \frac{\mathrm{r}_{2 \mathrm{n}} \mathrm{e}^{\mathrm{r}_{1 \mathrm{n}} \mathrm{t}}-\mathrm{r}_{1 \mathrm{n}} \mathrm{e}^{\mathrm{r}_{2 \mathrm{n}} \mathrm{t}}}{\mathrm{r}_{2 \mathrm{n}}-\mathrm{r}_{1 \mathrm{n}}}+\frac{\alpha}{\lambda} \frac{\mathrm{e}^{\mathrm{r}_{2 \mathrm{n}} \mathrm{t}}-\mathrm{e}^{\mathrm{r}_{1 \mathrm{n}} \mathrm{t}}}{\mathrm{r}_{2 \mathrm{n}}-\mathrm{r}_{1 \mathrm{n}}}\right] ; \quad \mathrm{t}>0 .
$$

Inverting Eq. (11) by means of the Fourier sine formulas we find that

$$
\begin{aligned}
\mathrm{u}(\mathrm{y}, \mathrm{z}, \mathrm{t})= & \frac{4 \mathrm{~V}}{\mathrm{~d}} \mathrm{H}(\mathrm{t}) \sum_{\mathrm{n}=1}^{\infty} \frac{\sin \left(\lambda_{N} \mathrm{z}\right)}{\lambda_{\mathrm{N}}} \mathrm{e}^{-\lambda_{N} \mathrm{y}}-\frac{8 \mathrm{~V}}{\pi \mathrm{d}} \mathrm{H}(\mathrm{t}) \sum_{\mathrm{n}=1}^{\infty} \frac{\sin \left(\lambda_{\mathrm{N}} \mathrm{z}\right)}{\lambda_{\mathrm{N}}} \\
& \times\left\{\int_{0}^{\infty} \frac{\mathrm{r}_{2 \mathrm{~N}} \mathrm{e}^{\mathrm{r}_{1 N} \mathrm{t}}-\mathrm{r}_{1 N} \mathrm{e}^{\mathrm{r}_{2 N} \mathrm{t}}}{\mathrm{r}_{2 \mathrm{~N}}-\mathrm{r}_{1 \mathrm{~N}}} \frac{\xi \sin (\mathrm{y} \xi)}{\xi^{2}+\lambda_{\mathrm{N}}^{2}} \mathrm{~d} \xi-\frac{\alpha}{\lambda} \int_{0}^{\infty} \frac{\mathrm{e}^{\mathrm{r}_{2 N} \mathrm{t}}-\mathrm{e}^{\mathrm{r}_{1 N} \mathrm{t}}}{\mathrm{r}_{2 \mathrm{~N}}-\mathrm{r}_{1 \mathrm{~N}}} \xi \sin (\mathrm{y} \xi) \mathrm{d} \xi\right\},
\end{aligned}
$$


in which $N=2 n-1$. Setting $d=2 h$ and changing the origin of the coordinate system at the middle of the channel, we get the following suitable form of the velocity field:

$$
\begin{aligned}
& \mathrm{u}(\mathrm{y}, \mathrm{z}, \mathrm{t})=\frac{2 \mathrm{~V}}{\mathrm{~h}} \mathrm{H}(\mathrm{t}) \sum_{\mathrm{n}=1}^{\infty} \frac{(-1)^{\mathrm{n}+1} \cos \left(\mu_{\mathrm{N}} \mathrm{z}\right)}{\mu_{\mathrm{N}}} \mathrm{e}^{-\mu_{\mathrm{N}} \mathrm{y}}-\frac{4 \mathrm{~V}}{\pi \mathrm{h}} \mathrm{H}(\mathrm{t}) \sum_{\mathrm{n}=1}^{\infty} \frac{(-1)^{\mathrm{n}+1} \cos \left(\mu_{\mathrm{N}} \mathrm{z}\right)}{\mu_{\mathrm{N}}} \\
& \times\left\{\int_{0}^{\infty} \frac{\mathrm{r}_{2 \mathrm{~N}} \mathrm{e}^{\mathrm{r}_{1 N} \mathrm{t}}-\mathrm{r}_{1 \mathrm{~N}} \mathrm{e}^{\mathrm{r}_{2 N} \mathrm{t}}}{\mathrm{r}_{2 \mathrm{~N}}-\mathrm{r}_{1 \mathrm{~N}}} \frac{\xi \sin (\mathrm{y} \xi)}{\xi^{2}+\mu_{\mathrm{N}}^{2}} \mathrm{~d} \xi-\frac{\alpha}{\lambda} \int_{0}^{\infty} \frac{\mathrm{e}^{\mathrm{r}_{2 N \mathrm{~N}}}-\mathrm{e}^{\mathrm{r}_{1 N} \mathrm{t}}}{\mathrm{r}_{2 \mathrm{~N}}-\mathrm{r}_{1 \mathrm{~N}}} \xi \sin (\mathrm{y} \xi) \mathrm{d} \xi\right\},
\end{aligned}
$$

with $\mu_{\mathrm{N}}=(2 \mathrm{n}-1) \pi /(2 \mathrm{~h})$. The last term of Eq. (13) is absent in [1, Eq. (16)]. Consequently, the results obtained in [1] are incomplete and these are corrected here. The non-trivial shear stresses $\tau_{1}(y, z, t)=S_{x y}(y, z, t)$ and $\tau_{2}(\mathrm{y}, \mathrm{z}, \mathrm{t})=\mathrm{S}_{\mathrm{xz}}(\mathrm{y}, \mathrm{z}, \mathrm{t})$ can be obtained introducing $\mathrm{u}(\mathrm{y}, \mathrm{z}, \mathrm{t})$ into Eqs. (20) and (21) from [1].

Of course, making $\lambda$ or $\lambda_{\mathrm{r}} \rightarrow 0$ in Eq. (13), the solutions corresponding to the second grade and Maxwell fluids performing the same motion are obtained. The velocity field $\mathrm{u}(\mathrm{y}, \mathrm{z}, \mathrm{t})$ corresponding to second grade fluids, for instance, takes the simple form

$$
\begin{aligned}
\mathrm{u}(\mathrm{y}, \mathrm{z}, \mathrm{t})= & \frac{2 \mathrm{~V}}{\mathrm{~h}} \mathrm{H}(\mathrm{t}) \sum_{\mathrm{n}=1}^{\infty} \frac{(-1)^{\mathrm{n}+1} \cos \left(\mu_{\mathrm{N}} \mathrm{z}\right)}{\mu_{\mathrm{N}}} \mathrm{e}^{-\mu_{\mathrm{N}} \mathrm{y}} \\
& -\frac{4 \mathrm{~V}}{\pi \mathrm{h}} \mathrm{H}(\mathrm{t}) \sum_{\mathrm{n}=1}^{\infty} \frac{(-1)^{\mathrm{n}+1} \cos \left(\mu_{\mathrm{N}} \mathrm{z}\right)}{\mu_{\mathrm{N}}} \int_{0}^{\infty} \frac{\xi \sin (\mathrm{y} \xi)}{\left(\xi^{2}+\mu_{\mathrm{N}}^{2}\right)\left[1+\alpha\left(\xi^{2}+\mu_{\mathrm{N}}^{2}\right)\right]} \exp \left(-\frac{v\left(\xi^{2}+\mu_{\mathrm{N}}^{2}\right) \mathrm{t}}{1+\alpha\left(\xi^{2}+\mu_{\mathrm{N}}^{2}\right)}\right) \mathrm{d} \xi .
\end{aligned}
$$

\section{Limiting case $h \rightarrow \infty$ (flow over a flat plate)}

In the absence of the side walls, namely when h goes to infinity in Eq. (13), the solution corresponding to the first problem of Stokes

$$
\mathrm{u}(\mathrm{y}, \mathrm{t})=\operatorname{VH}(\mathrm{t})\left\{1-\frac{2}{\pi} \int_{0}^{\infty} \frac{\mathrm{r}_{2} \mathrm{e}^{\mathrm{r}_{1} \mathrm{t}}-\mathrm{r}_{1} \mathrm{e}^{\mathrm{r}_{2} \mathrm{t}}}{\mathrm{r}_{2}-\mathrm{r}_{1}} \frac{\sin (\mathrm{y} \xi)}{\xi} \mathrm{d} \xi+\frac{2 \alpha}{\lambda \pi} \int_{0}^{\infty} \frac{\mathrm{e}^{\mathrm{r}_{2} \mathrm{t}}-\mathrm{e}^{\mathrm{r}_{1} \mathrm{t}}}{\mathrm{r}_{2}-\mathrm{r}_{1}} \xi \sin (\mathrm{y} \xi) \mathrm{d} \xi\right\}
$$

where $\mathrm{r}_{1}, \mathrm{r}_{2}=\frac{-\left(1+\alpha \xi^{2}\right) \pm \sqrt{\left(1+\alpha \xi^{2}\right)^{2}-4 v \lambda \xi^{2}}}{2 \lambda}$, is obtained. Note that Eq. (15) is in accordance with the result from [2, Eq. (7)]. The corresponding solution for second grade fluid is

$$
\mathrm{u}(\mathrm{y}, \mathrm{t})=\mathrm{VH}(\mathrm{t})\left[1-\frac{2}{\pi} \int_{0}^{\infty} \frac{\sin (\mathrm{y} \xi)}{\xi\left(1+\alpha \xi^{2}\right)} \exp \left(-\frac{v \xi^{2} \mathrm{t}}{1+\alpha \xi^{2}}\right) \mathrm{d} \xi\right] .
$$

The above expression is also identical to the equality (3) of [3].

\section{References}

1. Fetecau, C., Hayat, T., Khan, M., Fetecau, C.: Unsteady flow of an Oldoyd-B fluid induced by the impulsive motion of a plate between two side walls perpendicular to the plate. Acta Mech. 198, 21-33 (2008)

2. Christov, C.I., Jordan, P.M.: Comment on "Stokes' first problem for an Oldroyd-B fluid in a porous half space" [Phys. Fluids 17, 023101 (2005)]. Phys. Fluids 21, 069101-069102 (2009)

3. Christov, I.C., Christov, C.I.: Comment on "On a class of exact solutions of the equations of motion of a second grade fluid" by C. Fetecau and J. Zierep (Acta Mech. 150, 135-138, 2001), Acta Mech. doi:10.1007/s00707-010-0300-2 